\title{
Intensity Modulated Versus 3D-Conformal Radiotherapy in the Treatment of Locally Advanced Cervical Cancer: a Dosimetric study
}

\author{
Rasha Salama ${ }^{1}$ Hamdy Zawam ${ }^{1}$, Mamdouh Haggag ${ }^{1}$, Wael Edesa ${ }^{1}$, Mostafa Abdel Moez², \\ Mohamed M. Eltoukhy ${ }^{3}$, Walid Abdel Moneiem ${ }^{4}$, Mostafa El-Haddad ${ }^{1}$
}

${ }^{1}$ Department of Clinical Oncology, Faculty of Medicine, Cairo University, Egypt, ${ }^{2}$ Department of Radiation Physics, Faculty of Medicine, Cairo University, Egypt, ${ }^{3}$ Department of Radiodiagnosis, Faculty of Medicine, Cairo University, Egypt, ${ }^{4}$ Department of Clinical oncology, Ain Shams University, Egypt.

Background and Aim: Three dimensional conformal radiotherapy (3D-CRT) is considered the standard technique used in cervical cancer. However, toxicity is still a concern. The aim of this study was to compare 3D-CRT with intensity modulated radiotherapy (IMRT) as a way to reduce toxicity.

Material and Methods: Thirty patients with confirmed diagnosis of cervical cancer stage IIB-IVA treated with concurrent chemo-radiation using 3D-CRT were re-planned using IMRT. Analysis was done for target coverage, organ at risk, as well as dose homogeneity. Planning was done on Xio-Elekta planning system.

Results: $\mathrm{D}_{95} \%$ was $42.7 \mathrm{~Gy}$ for 3D-CRT plans versus $41 \mathrm{~Gy}$ for IMRT plans and V40 for the rectum, bladder and small bowel was significantly less for IMRT. For the femoral heads: V30 was $59.4 \%$ with IMRT versus $96 \%$ with 3D-CRT. V10 of the pelvic bone marrow was significantly less with 3D-CRT than IMRT; however V20 was better for IMRT plans. In terms of overall treatment time, IMRT calculated treatment time is longer, with mean integral monitor unit (IMU) of 1177.01 compared to 144.30 with 3D-CRT.

Conclusion: IMRT for locally advanced cervical cancer can reduce the dose to risk structures without compromising tumour coverage.

Key words: Cervical Cancer; 3D-CRT; IMRT; Organs at Risk; Target Definition. Corresponding Author: Mostafa El-Haddad M.D., FRCR., HMD.

E-mail: mostafa.haddad@kasralainy.edu.eg

\section{INTRODUCTION}

Intensity modulated radiotherapy (IMRT) is used with great success in the treatment of head and neck as well as prostate cancers ${ }^{1-3}$. This success was in both dose escalation as well as reduction of toxicity.

In cervical cancer, concurrent chemo-radiation is considered the standard of care in patients with advanced stage (stage IIB and above), with many patients can survive more than five years which may allow for the emergence of treatment related toxicity. The use of external beam radiotherapy for whole pelvis irradiation exposes large part of the intestine, rectum, and bladder to unnecessary radiation. Reducing the dose to risk structures may improve tolerance to chemotherapy and decrease treatment related interruption. Early experience with IMRT in cervical cancer is very promising ${ }^{4,5}$.

The aim of this work was to study the value of IMRT compared to 3 dimensional conformal radiotherapy (3D-CRT) in the management of cervical cancer and its potential benefit in reducing the dose and side effects to risk structures.

\section{PATIENTS AND METHODS}

Thirty patients with confirmed diagnosis of cervical carcinoma were recruited for this study between 2010 and 2012 in the Department of Clinical Oncology, Cairo University Hospitals, Egypt.

\section{Radiotherapy Technique}

Target volume and risk structures were delineated according to the GYN IMRT consortium ${ }^{6}$, with the clinical target volume (CTV) including the uterine body, upper vagina, parametria and proximal uterosacral ligaments (the whole uterosacral ligaments if involved) in all patients. For lymph nodes, it was delineated $6 \mathrm{~mm}$ around the vessels starting at the bifurcation of the common iliac vessels then around the external and internal iliac vessels. Dose prescribed for all was 45 Gy/25 treatment $/ 5$ weeks with concomitant weekly cisplatin $40 \mathrm{mg} / \mathrm{m} 2$. This was followed by low dose rate (LDR) brachytherapy with $30 \mathrm{~Gy}$ prescribed to point A. Planning target volume (PTV) was taken with a $1 \mathrm{~cm}$ margin around the CTV. 


\section{Comparison of treatment techniques}

We compared the 3D-CRT four field box technique, the actual treatment technique (Figure 1), with full inverse based IMRT optimization plans. Both plan sets were generated on Xio (Elekta, UK) for an Elekta Precise linear accelerator with a multileaf collimator. The leaf width was $1 \mathrm{~cm}$ at the isocenter and the beam energy was $15 \mathrm{MV}$. The dose calculation was done using superposition algorithm with calculation grid size of $2 \mathrm{~mm}$.

The treatment times were measured delivering the treatment on an Elekta-Precise machine using a dose rate of $600 \mathrm{MU} / \mathrm{min}$.

\section{IMRT technique}

Seven isocentric co-planner equi-spaced fields using $15 \mathrm{MV}$ beam (only used for plan comparison not for actual treatment) were used. Beam angles used were 180, 232, 248, 336, 28, 80, and 132, (Figure 2). The maximum number of iteration was set as 100 . Segmentation parameters were set to a minimum segment size of $2 \mathrm{~cm}$ and maximum intensity level of 10 .

\section{D-CRT Comparative evaluation of plans}

Evaluation was performed for a total dose of $45 \mathrm{~Gy}$ prescribed/ delivered as the mean dose to the PTV for both the 3D-CRT and the IMRT plan for each patient. This prescription mode facilitated comparison of the homogeneous plans with the more inhomogeneous IMRT plans. The 3D-CRT and IMRT plans were then compared with respect to target volume coverage, the dose to the organs at risk and dose homogeneity.

\section{RESULTS}

The mean age of the patients was 50 years, with majority of cases $(90 \%)$ had squamous cell carcinoma pathology. According to the International Federation of Gynecology and Obstetrics (FIGO) staging system, $56.6 \%$ had stage 2 disease. Haemoglobin level was reduced in nine (30\%) patients (range: $7.5-9 \mathrm{~g} / \mathrm{dl}$ ) during chemo-radiotherapy with $23.3 \%$ required blood transfusion. Baseline patient's characteristics are summarized in Table 1.

The median duration of the whole pelvic radiotherapy was 5 weeks (range: 4.5-7). Patients started phase 2 treatment (LDR brachytherapy) after 2 weeks (range: 2- 4) of ending pelvic radiotherapy with median dose of $25 \mathrm{~Gy}$ prescribed to point A (range: $20-30$ ). Fourteen patients (46\%) received parametrial boost with mean dose of $15 \mathrm{~Gy}$ (range: 10- 20).

\section{PTV Coverage}

Dose distribution in Figure 3 and 4, shows clearly the difference between 3D-CRT and IMRT in the high dose region with IMRT plan can spare posterior part of the rectum as well as anterior part of the bladder. Table 2 provides an overview of the relevant plan parameters (i.e., the mean, median, and partial-volume doses to the rectum, bladder and femurs).

The $\mathrm{D}_{95} \%, \mathrm{D}_{\max }, \mathrm{D}_{\text {min }}$ and $\mathrm{D}_{\text {mean }}$ for 3D-CRT compared to IMRT shown in Table 2 illustrates that there is a minimal difference between 3D-CRT and IMRT as regard the PTV coverage; however there was a difference in homogeneity index for conformal radiotherapy (1.23) versus IMRT (1.12).

\section{Organs at risk}

For each individual patient, IMRT consistently reduced the maximal dose to the rectum, bladder, as well as femurs.

V40 for the rectum, bladder and small bowel was significantly less with IMRT. In femoral heads, the V30 was $59.4 \%$ versus $96 \%$ with IMRT and 3D-CRT respectively. V10 of the pelvic bone marrow was significantly less with 3D-CRT than IMRT; however V20 was better for IMRT plans. In terms of overall treatment time, IMRT calculated treatment time is longer, with mean integral monitor unit (IMU) of 1177.01 compared with 144.30 for 3D CRT Table 3. 


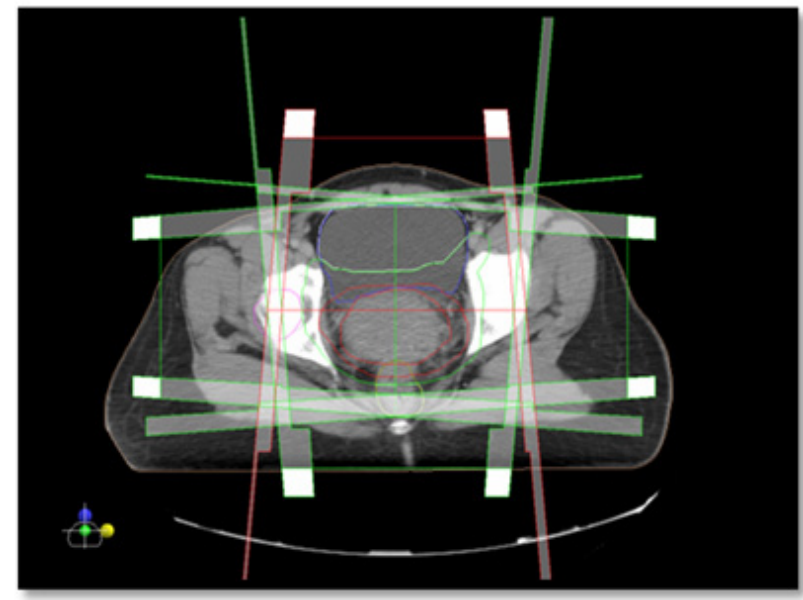

Figure 1: The beam arrangement in 4 fields whole pelvis box technique with 3 dimensional conformal radiotherapy

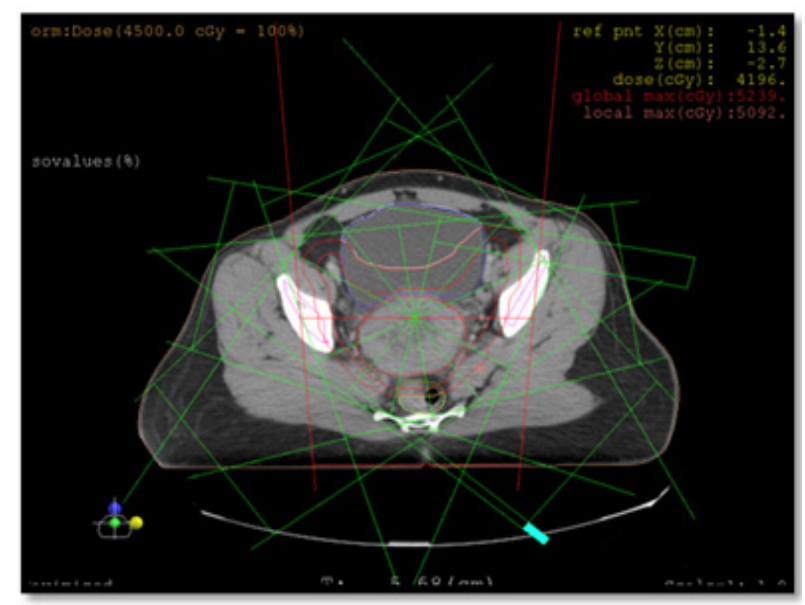

Figure 2: The beam arrangement in 7 fields Intensity modulated radiotherapy technique.

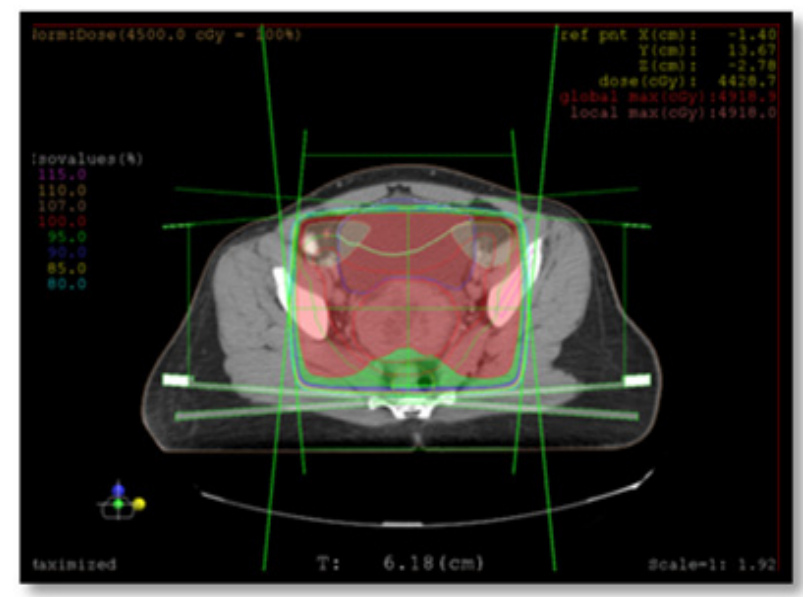

Figure 3: Dose distribution in 3D-CRT

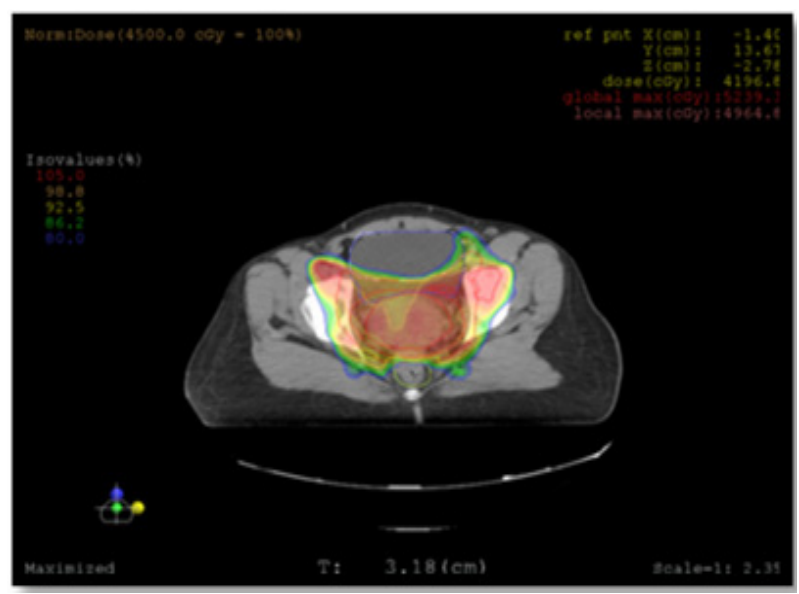

Figure 4: Dose distribution in IMRT (Axial view)

Table 1: Baseline patients' characteristics

\begin{tabular}{lc}
\hline Characteristics & No. (\%) \\
\hline Age (years) & $50(38-65)$ \\
Median (range) & $8(26)$ \\
\hline Multiple marriages & \\
\hline Pathological type & $24(80)$ \\
Non keratinized SCC $\dagger$ & $3(10)$ \\
Keratinized SCC & $3(10)$ \\
Adenocarcinoma & $17(56.6)$ \\
\hline FIGO staging ${ }^{* *}$ & $9(30)$ \\
Stage II & $4(13.4)$ \\
Stage III & \\
Stage IV A & $11.5(8.6-12.5)$ \\
\hline Hemoglobin (g/dl) & \\
Median (range) & \\
\hline
\end{tabular}

"SCC: Squamous cell carcinoma; ${ }^{* *}$ FIGO: International federation of Gynaecology and obstetrics 
Kasr-El-Aini Journal Of Clinical Oncology And Nuclear Medicine

Vol. $11 \mid$ No. $1-2$ 2015

IMRT vs. 3D-CRT in Cervical Cancer

Table 2: Dosimetric comparison between mean parameters for WP-3D-CRT and WP-IMRT

\begin{tabular}{lcc}
\hline Parameter & 3D-CRT(SD) & IMRT(SD) \\
\hline $\mathbf{D}_{\mathbf{9 5}}(\mathbf{\%})$ & $95.67 \%( \pm 1.4)$ & $93.22 \%( \pm 1.67)$ \\
\hline $\mathbf{D}_{\max }$ & $107.07 \%( \pm 1.44)$ & $110 \%( \pm 2.16)$ \\
\hline $\mathbf{D}_{\min }$ & $90.80( \pm 3.00)$ & $90.20( \pm 4.11)$ \\
\hline $\mathbf{D}_{\text {mean }}$ & $100.25 \%( \pm 1.04)$ & $100.3 \%( \pm 0.134)$ \\
\hline $\mathbf{H I}$ & 1.23 & 1.12 \\
\hline $\mathbf{C I}$ & $1.3 \% \pm 0.2$ & $1.4 \pm 0.2$ \\
\hline
\end{tabular}

WP: whole pelvis; IMRT=intensity-modulated radiotherapy; $3 \mathrm{D}-\mathrm{CRT}=$ three-dimensional conformal radiotherapy; $\mathrm{HI}=$ homogeneity index; $\mathrm{CI}$ : Confidence interval.

Data presented as mean, min, and maximum, dose \pm standard deviation to target,

Table 3: Clinically relevant plan parameter. Doses to organs at risk for 3D-CRT and IMRT

\begin{tabular}{|c|c|c|c|}
\hline Organ & Parameter & $\begin{array}{c}\text { Mean dose }( \pm \text { SD }) \\
\text { 3D-CRT }\end{array}$ & $\begin{array}{c}\text { Mean dose }( \pm \text { SD }) \\
\text { IMRT }\end{array}$ \\
\hline \multirow{2}{*}{ Rectum } & V40 & $78.48 \%( \pm 16.04)$ & $37.95 \%( \pm 11.59)$ \\
\hline & V45 & $19.57 \%( \pm 17.38)$ & $14.47 \%( \pm 7.54)$ \\
\hline \multirow{2}{*}{ Bladder } & V40 & $88.45 \%( \pm 12.67)$ & $63.52( \pm 24.39)$ \\
\hline & V45 & $69.29 \%( \pm 22.06)$ & $33.48 \%( \pm 23.07)$ \\
\hline \multirow{2}{*}{ Small Bowel } & $\mathrm{V} 40$ & $26.84 \%( \pm 11.35)$ & $16.67 \%( \pm 9.01)$ \\
\hline & V45 & $17.87 \%( \pm 8.69)$ & $6 \%( \pm 8.3)$ \\
\hline \multirow{2}{*}{ Femoral head } & $\mathrm{V} 30$ & $96.09 \%( \pm 9.91)$ & $59.40 \%( \pm 16.23)$ \\
\hline & $\mathrm{V} 20$ & $11.30 \%( \pm 10.88)$ & $23.29 \%( \pm 16.19)$ \\
\hline \multirow{2}{*}{ Pelvic Bone marrow } & V10 & $92.46 \%( \pm 10.14)$ & $98.37 \%( \pm 1.71)$ \\
\hline & $\mathrm{V} 20$ & $89.91 \%( \pm 6.66)$ & $84.31 \%( \pm 10.20)$ \\
\hline
\end{tabular}

\section{DISCUSSION}

Phase III randomized trials and meta analysis showed clearly that concurrent chemo-radiation is the standard of care for patients with inoperable squamous cell carcinoma of the cervix uteri ${ }^{7-10}$. Concurrent chemo-radiation is effective but it is not without toxicity ${ }^{11-13}$. With $3 \mathrm{D}-\mathrm{CRT}$ it may be impossible to reduce the dose to organs in close proximity to the target, while IMRT is used to shape the dose around the risk organs without compromising the coverage of the tumour and it was used successfully in many pelvic tumours including prostate, rectum, as well as anal canal ${ }^{14-16}$.

Hoskin et al, ${ }^{17}, 18$ showed that pre-treatment haemoglobin level and peripheral blood lymphocyte count may be an important prognostic factor in patients with cervical cancer patients, a similar finding to others $^{19-21}$ which may reflect the importance of trying to save as much red marrow as possible. Brent et al tested associations between hematologic nadirs during chemoradiotherapy and the volume of bone marrow receiving $\geq 10$ and 20 Gy (V10 and V20) using a linear regression model and they found significant negative correlations between white blood cell count nadir and $\mathrm{V} 10$ and $\mathrm{V} 20^{22}$. Patients with V10 $\geq 95 \%$ were more likely to experience grade 3 leukopenia than were patients with V20 $>76 \%$. In our patients' cohort, V10 of the pelvic bone marrow was less in 3D-CRT plans than IMRT; however V20 was better for IMRT plans. We did not add a strong dose constraint for the bone marrow in IMRT plans to avoid under coverage of the PTV and to see the effect of low dose radiation on the pelvic bone marrow. Mell et al studied the effect of low dose radiotherapy to the bone marrow in 20 patients with anal canal squamous cell carcinoma and showed on multiple regression analysis, that increased pelvic bone marrow V5, V10, V15 and V20 were associated significantly with decreased white blood cell count and nadirs $^{23}$. So it may be helpful to keep the dose to the pelvic marrow as low as possible.

IMRT technique allows irradiating pelvic lymph node with minimal exposure to the intestine ${ }^{24}$. In our study, the dose to intestinal bag reduced significantly from $17 \%$ to $6 \%$ for the high dose region, this may help to reduce the toxicity and continuation of treatment without any interruption. However, the PTV margin in our study was taken as $1 \mathrm{~cm}$ from the CTV, to simulate that in 3D-CRT. But if we put into consideration the large and complex day-to-day tissue variations in the pelvic area we may have to add a larger margin of 2 or $3 \mathrm{~cm}$ to avoid any serious under coverage that may limit 


Vol. $11 \mid$ No. $1-2 \quad 2015 \quad$ Salama et al

the efficacy of IMRT ${ }^{25,26}$. Bladder-filling variations can have a large impact on shape and position of the cervixuterus. Drinking instructions, used to minimize bladderfilling variations, may have limited efficacy ${ }^{27}$. This movement variability may not be of great importance with 3D-CRT as the volume treated includes the whole pelvis without differentiation between risk structures and target, however exposure of the intestine in case of empty bladder will be a concern. It is recommended to re-plan patients at least once weekly or to use image guidance on the machine to modify the internal target volume (ITV) which allow for this movement.

In conclusion, IMRT may be of great value for the treatment of cervical cancer; however image guidance should be used to ensure adequate tumour coverage.

\section{REFERENCES}

1. Marta GN, Silva V, de Andrade Carvalho H, et al. Intensity-modulated radiation therapy for head and neck cancer: systematic review and meta-analysis. Radiother Oncol. 2014;110:9-15.

2. Zong J, Lin S, Lin J, et al. Impact of intensity-modulated radiotherapy on nasopharyngeal carcinoma: Validation of the 7th edition AJCC staging system. Oral Oncol. 2015;51:254-259.

3. Eminowicz G, Dean C, Shoffren O, et al. Intensitymodulated radiotherapy (IMRT) to prostate and pelvic nodes-is pelvic lymph node coverage adequate with fiducial-based image-guided radiotherapy? Br J Radiol, 2014;87:20130696.

4. Portelance L, Chao KS, Grigsby PW, et al. Intensitymodulated radiation therapy (IMRT) reduces small bowel, rectum, and bladder doses in patients with cervical cancer receiving pelvic and para-aortic irradiation. Int J Radiat Oncol Biol Phys. 2001;51:261-266.

5. Reddy NM, Mazur AK, Sampath S, et al. The potential for dose dumping in normal tissues with IMRT for pelvic and H\&N cancers. Med Dosim. 2008;33:55-61.

6. Lim K, Small W Jr, Portelance L, et al. Consensus guidelines for delineation of clinical target volume for intensity-modulated pelvic radiotherapy for the definitive treatment of cervix cancer. Int J Radiat Oncol Biol Phys. 2011;79:348-355.

7. Stehman FB. Concurrent chemoradiation in carcinoma of the uterine cervix. Semin Oncol, 1992;19:88-91.

8. Tseng CJ, Chang CT, Lai CH, et al. A randomized trial of concurrent chemoradiotherapy versus radiotherapy in advanced carcinoma of the uterine cervix. Gynecol Oncol. 1997;66:52-58.

9. Eifel PJ. Chemoradiation for carcinoma of the cervix: advances and opportunities. Radiat Res. 2000;154:229236.

10. Datta NR and Agrawal S. Does the evidence support the use of concurrent chemoradiotherapy as a standard in the management of locally advanced cancer of the cervix, especially in developing countries? Clin Oncol (R Coll Radiol). 2006;18:306-312.
11. Grigsby PW. Rectal complications in patients with carcinoma of the cervix: the concept of a rectal reference dose. Int J Radiat Oncol Biol Phys. 1994;28:1271-1272.

12. Suggs CL 3rd, Lee JC, Lewis GC, et al. Advanced cervical cancer therapy: concurrent radiation therapy and cisplatin chemotherapy for advanced cervical cancer--a toxicity report. Am J Clin Oncol. 1989;12:461-466.

13. Okuhata Y, Yasukochi H, Sugiyama T, et al. [Injuries of the sigmoid colon following radiation therapy of carcinoma of the uterine cervix--a method of estimating the radiation dose to the sigmoid colon]. Nihon Igaku Hoshasen Gakkai Zasshi. 1990;50:262-270.

14. $\mathrm{Ng} \mathrm{M}$, Leong $\mathrm{T}$, Chander $\mathrm{S}$, et al. Australasian Gastrointestinal Trials Group (AGITG) contouring atlas and planning guidelines for intensity-modulated radiotherapy in anal cancer. Int J Radiat Oncol Biol Phys. 2012;83:1455-1462.

15. Nutting CM, Convery DJ, Cosgrove VP, et al. Reduction of small and large bowel irradiation using an optimized intensity-modulated pelvic radiotherapy technique in patients with prostate cancer. Int J Radiat Oncol Biol Phys. 2000;48:649-656.

16. Arbea L, Ramos LI, Martínez-Monge R, et al. Intensitymodulated radiation therapy (IMRT) vs. 3D conformal radiotherapy (3D-CRT) in locally advanced rectal cancer (LARC): dosimetric comparison and clinical implications. Radiat Oncol. 2010;5:17.

17. Vissink A, van Luijk P, Langendijk JA, et al. Current ideas to reduce or salvage radiation damage to salivary glands. Oral Dis. 2015;21:e1-e10.

18. Hoskin PJ, Rojas AM, Peiris SN, et al. Pre-treatment haemoglobin and peripheral blood lymphocyte count as independent predictors of outcome in carcinoma of cervix. Clin Oncol (R Coll Radiol). 2014;26:179-184.

19. Barkati M, Fortin I, Mileshkin L, et al. Hemoglobin level in cervical cancer: a surrogate for an infiltrative phenotype. Int J Gynecol Cancer. 2013;23:724-729.

20. Gupta S, Singh PK, Bisth SS, et al. Role of recombinant human erythropoietin in patients of advanced cervical cancer treated "by chemoradiotherapy". Cancer Biol Ther. 2009;8:13-17.

21. Marchal C, Rangeard L, and Brunaud C. [Anemia impact on treatments of cervical carcinomas]. Cancer Radiother. 2005;9:87-95.

22. Rose BS, Aydogan B, Liang Y, et al. Normal tissue complication probability modeling of acute hematologic toxicity in cervical cancer patients treated with chemoradiotherapy. Int $\mathrm{J}$ Radiat Oncol Biol Phys. 2011;79:800-807.

23. Mell LK, Tiryaki H, Ahn KH, et al. Dosimetric comparison of bone marrow-sparing intensity-modulated radiotherapy versus conventional techniques for treatment of cervical cancer. Int J Radiat Oncol Biol Phys. 2008;71:1504-1510.

24. Vargo JA, Kim H, Choi S, et al., Extended Field Intensity Modulated Radiation Therapy With Concomitant Boost for Lymph Node-Positive Cervical Cancer: Analysis of Regional Control and Recurrence Patterns in the Positron Emission Tomography/Computed Tomography Era. Int J Radiat Oncol Biol Phys. 2014;90:1091-1098. 
25. Bondar L, Hoogeman M, Mens JW, et al. Toward an individualized target motion management for IMRT of cervical cancer based on model-predicted cervix-uterus shape and position. Radiother Oncol. 2011;99:240-245.

26. Buchali A, Koswig S, Dinges S, et al. Impact of the filling status of the bladder and rectum on their integral dose distribution and the movement of the uterus in the treatment planning of gynaecological cancer. Radiother Oncol. 1999;52:29-34.

27. Ahmad R, Bondar L, Voet P, et al. A margin-of-theday online adaptive intensity-modulated radiotherapy strategy for cervical cancer provides superior treatment accuracy compared to clinically recommended margins: a dosimetric evaluation. Acta Oncol. 2013;52:1430-1436. 